\title{
Paradoxes in thyroid carcinoma treatment: analysis of the SEER database 2010-2013
}

\author{
Ping Zhou ${ }^{1}$, Shuangming Tian ${ }^{1}$, Jiale $\mathrm{Li}^{1}$, Yongfeng Zhao ${ }^{1}$, Wengang Liu ${ }^{1}$, Yan \\ Zhang ${ }^{1}$, Zheyu Hu${ }^{1,2}$ \\ ${ }^{1}$ Department of Ultrasound, the Third Xiangya Hospital of Central South University, China \\ ${ }^{2}$ Information Security and Big Data Institution, Central South University, China \\ Correspondence to: Zheyu Hu, email: zheyuhu@yahoo.com
}

Keywords: thyroid carcinoma, epidemiology and end results (SEER) database, surgery and radiation treatment, cox regression model, propensity score

Received: October 04, $2016 \quad$ Accepted: November 09, $2016 \quad$ Published: November 16, 2016

\section{ABSTRACT}

Thyroid cancer is a common malignant disease with high survival rate $(98.1 \%$, 2006-2012, Surveillance Epidemiology and End Results (SEER) program). In this study, we investigated the treatment paradoxes in thyroid TO and micro-carcinoma patients. 48,234 thyroid carcinoma patients were identified from 2010 to 2013 in SEER*Stat database (version 8.2.1) released in 2016. Survival analysis showed a significantly lower thyroid carcinoma-specific survival in TO patients compared with T1-T3 patients. In propensity score analysis, T0 patients had a similar survival curve with T1-T3 patients when lymph node and distant metastasis stages were matched. When all variables, including radiation and surgery treatment, were matched, T0 patients had significantly higher survival compared to T3 patients. These findings suggested that more metastasis and less treatment led to poorer prognosis in T0 patients. Another paradox is about thyroid micro-carcinoma. The survival rate of micro-carcinoma patients was high (4 years survival rate was $99.92 \%$ ), and more than $99 \%$ micro-carcinoma patients received surgery. Interestingly, all the patients who died because of thyroid carcinoma received surgery. Survival analysis showed no difference in survival when patients stratified by surgery or radiation. In conclusion, we suggested paradoxes in thyroid carcinoma treatment: over-treated in microcarcinoma patients and less-treated in T0 patients.

\section{INTRODUCTION}

Thyroid carcinoma is a kind of head and neck malignant disease with increasing incidence rate [1] and high overall survival rate [2]. According to National Comprehensive Cancer Network (NCCN) guidelines, the first diagnosis step of thyroid nodules is to measure serum thyroid-stimulating hormone (TSH) level and to do ultrasound of the thyroid and central neck. Fine-needle aspiration (FNA) with biopsy examination is required for the histology confirmation.

According to American Joint Committee on Cancer (AJCC) TNM staging for thyroid cancer (7th ed., 2010), thyroid tumors are staged based on the primary tumor size. T0 patients have no evidence of primary malignant tumor. But diagnosis can be confirmed by radioiodine imaging or FNA biopsy in metastasis sites. T1 tumors are less than $2 \mathrm{~cm}$. T2 tumors are between $2 \mathrm{~cm}$ and $4 \mathrm{~cm}$. T3 tumors are more than $4 \mathrm{~cm}$ but limited to thyroid or any tumor size with minimal extrathyroid extension. T4 tumors extend beyond the thyroid capsule to invade subcutaneous sift tissues or prevertebral fascia. T0 patients have no evidence of primary malignant thyroid tumor, but diagnosis can be made based on histological and cytological confirmation in metastasis sites.

Total lobectomy alone is recommended for patients with thyroid micro-carcinoma (T1a). Postoperative radioiodine is recommended for $\mathrm{T} 1 \mathrm{~b}-\mathrm{T} 4$ patients. For T0 patients, no local surgery or radiation treatment was recommended. Focal papillary carcinoma (tumor size $<4 \mathrm{~cm}$ in diameter) with no prior radiation exposure, no lymph node metastases, and no extrathyroidal 
extension, lobectomy is recommended. Otherwise, complete thyroidectomy is recommended [3]. Currently, compared to unilateral lobectomy, aggressive completion thyroid resection is preferable, because it reduce the local recurrence and nodal metastasis [4]. One exception is T0 tumor. In previous study, T0 tumors have been perceived to have a low mortality rate and are featured as well-differentiated [5]. So, compared to T1-T3 patients, T0 tumors are less treated for a long term. The clinical investigations for $\mathrm{T} 0$ patients are rare, too.

On the other hand, T1a thyroid micro-carcinoma (tumor size less than $1 \mathrm{~cm}$ in diameter) is widely investigated and aggressively treated. Though lobectomy alone is recommended for thyroid micro-carcinoma [6], surgeons always decide to perform completion thyroidectomy in most clinical settings (74.3\%) [7]. Recently, to eliminate severe complications and extra cost after aggressive treatment, some specialists began to oppose completion thyroidectomy and advocate unilateral lobetectomy [8-9]. Oncologists also recommend nonsurgical treatment, such as ultrasound-guided thermal laser ablation (LA), for micro-carcinoma [10-11].

The SEER program of the National Cancer Institute (NCI) is a population-based cancer registry covering approximately $30 \%$ of the population in the United States. This database is the largest publicly available and authoritative information source on cancer incidence and survival. Using this reliable and large-scale research dataset, we gathered information of 48,243 thyroid carcinoma patients diagnosed in 2010-2013. SEER database collected full information about the thyroid carcinoma $\mathrm{T}$ staging based on 7 th edition of AJCC staging system.

\section{RESULTS}

\section{Demographic and clinical features}

Of 48,243 patients included in this study, 47,360 cases had definite AJCC 7th T stage record. 84 patients were in T0 stage, 28,067 patients were in T1 stage, 7,727 patients were in T2 stage, 9,375 patients were in T3 stage, and 2,107 were in T4 stage. The mean (SD) and median (interquartile range) age for each $\mathrm{T}$ stage was listed in Table 1. ROC curve analysis determined that age of diagnosis at $59 \mathrm{yr}$ was the optional cutoff age that maximizes sensitivity and specificity for predicting both thyroid carcinoma-specific mortality and all-cause mortality (Supplementary Figure S1 and S2). No racial disparity was observed among $\mathrm{T}$ stages.

The mean (SD) survival months for each $\mathrm{T}$ stage were also listed in Table 1. T4 patients had significantly shorter survival months than other patients. Chi-square tests or Fisher's exact tests $(n<5)$ demonstrated that T4 patients had the highest all-cause mortality rate and thyroid carcinoma-specific mortality rate. Interestingly, both the all-cause mortality rate and thyroid carcinoma-specific mortality rate in $\mathrm{T} 0$ patients were significantly higher than T1-T3 patients (Table 1). Possible reasons might be that T0 patients were older than T1 to T3 patients, and had higher proportion of poorly differentiated tumor (12.5\%), lymph node metastasis $(77.2 \%)$ and distant metastasis $(28.6 \%)$ than T1-T3 patients. T0 patients seemed to have a more progressive carcinoma than $\mathrm{T} 1-\mathrm{T} 3$ patients, but less T0 patients received any type of radiation or surgery (55.56\% and $34.52 \%$ respectively) than $\mathrm{T} 1-\mathrm{T} 3$ patients (Table 1).

\section{Survival analysis stratified by $T$ stages}

Among 47,360 cases with definite T stage records, 1,568 all-cause deaths and 703 thyroid carcinomaspecific deaths were observed. 1-, 2- and 4-year estimated thyroid carcinoma-specific survival rates were $98.60 \%$, $98.26 \%$ and $98.03 \%$, respectively (Supplementary Figure S3B). Compared to T1-T3 patients, T0 patients had a poorer all-cause survival and thyroid carcinomaspecific survival. In T0 patients, the 1-, 2- and 4-year estimated survival rates were $95.12 \%, 88.02 \%$ and $88.02 \%$, respectively, as compared to $99.06 \%, 97.14 \%$ and $95.58 \%$, respectively, in T3 patients (Figure 1A and 1B, log-rank $P$-value $<0.001$, Supplementary Table S1). In T0 patients, the 1-, 2- and 4-year thyroid carcinoma specific estimated survival rates were $97.42 \%, 95.25 \%$ and $95.25 \%$, respectively, as compared to $99.38 \%, 98.96 \%$ and $98.48 \%$, respectively, in T3 patients (Figure 1C and 1D, log-rank $P$-value $<0.0001$, Supplementary Table S2).

\section{Risk factors for all-cause mortality and thyroid carcinoma-specific mortality}

Univariate Cox regression analyses showed that age, black race, TNM stage, differentiation grade, radiation and surgery treatment were significant risk factors of all-cause death and thyroid carcinoma-specific death. In multivariate Cox regression model, after controlling all other influential risk factors, T0 stage did not show significant risk for all-cause death compared to T1-T3 patients (Table 2, $p>0.1$ ). For thyroid carcinoma-specific death, multivariate Cox regression analysis showed that the risk of T0 patients was significantly higher than T1 patients ( $p=0.009$ by controlling other confounding factors in multivariate analysis, Table 2).

\section{Adjusting for patient characteristics using propensity score matching}

To account for potential bias due to an imbalance between $\mathrm{T} 0$ and $\mathrm{T} 1-\mathrm{T} 1$ patients regarding the age, race, tumor differentiation grade, $\mathrm{N}$ stage, $\mathrm{M}$ stage, surgery and radiation treatment, propensity score matching was carried out as described in Methods. In survival analysis, 
Table 1: Characteristics for patients with T0-T4

\begin{tabular}{|c|c|c|c|c|c|c|}
\hline \multirow{2}{*}{ Covariate } & \multirow{2}{*}{ level } & \multicolumn{5}{|c|}{ T stages } \\
\hline & & T0 $(n=84)$ & T1 $(n=28067)$ & T2 $(n=7727)$ & T3 $(n=9375)$ & T4 $(n=2107)$ \\
\hline Age & & $\begin{array}{c}55.12 \pm 17.84 \\
57(42.5,66.5)\end{array}$ & $\begin{array}{c}50.28 \pm 14.47 \\
51(40,61)\end{array}$ & $\begin{array}{c}47.16 \pm 16.12 \\
46(35,58)\end{array}$ & $\begin{array}{c}50.31 \pm 16.59 \\
50(38,62)\end{array}$ & $\begin{array}{c}61.33 \pm 17.64 \\
63(50,74)\end{array}$ \\
\hline Survival months & & $22.30 \pm 14.70$ & $21.52 \pm 14.09$ & $21.55 \pm 13.94$ & $21.50 \pm 13.95$ & $16.30 \pm 14.56$ \\
\hline \multirow[t]{2}{*}{ All-cause mortality } & No & $75(89.29 \%)$ & $27632(98.45 \%)$ & 7577 (98.06\%) & $9131(97.40 \%)$ & $1377(65.35 \%)$ \\
\hline & Yes & $9(10.71 \%)$ & $435(1.55 \%)$ & $150(1.94 \%)$ & $244(2.60 \%)$ & $730(34.65 \%)$ \\
\hline \multirow{2}{*}{$\begin{array}{l}\text { Thyroid carcinoma- } \\
\text { specific death }\end{array}$} & No & $75(96.15 \%)$ & $27632(99.91 \%)$ & $7577(99.61 \%)$ & $9131(99.07 \%)$ & $1377(71.02 \%)$ \\
\hline & Yes & $3(3.85 \%)$ & $25(0.09 \%)$ & $30(0.39 \%)$ & $86(0.93 \%)$ & $562(28.98 \%)$ \\
\hline \multirow[t]{3}{*}{ Race } & white & $69(82.14 \%)$ & $22981(81.88 \%)$ & $6215(80.43 \%)$ & $7379(78.71 \%)$ & $1671(79.31 \%)$ \\
\hline & black & $9(10.71 \%)$ & $1872(6.67 \%)$ & $576(7.45 \%)$ & $607(6.47 \%)$ & $128(6.07 \%)$ \\
\hline & other & $6(7.14 \%)$ & $3214(11.45 \%)$ & $936(12.11 \%)$ & $1389(14.82 \%)$ & $308(14.62 \%)$ \\
\hline \multirow[t]{4}{*}{ Grade } & Well-differentiated & $5(62.50 \%)$ & $6122(86.42 \%)$ & $1754(79.80 \%)$ & $1894(68.82 \%)$ & $219(20.58 \%)$ \\
\hline & Moderate & $2(25.00 \%)$ & $875(12.35 \%)$ & $373(6.97 \%)$ & $614(22.31 \%)$ & $116(10.90 \%)$ \\
\hline & $\begin{array}{c}\text { Poorly } \\
\text { differentiated }\end{array}$ & $1(12.50 \%)$ & $87(1.23 \%)$ & $71(3.23 \%)$ & $244(8.87 \%)$ & $153(27.52 \%)$ \\
\hline & undifferentiated & $0(0.00 \%)$ & $0(0.00 \%)$ & $0(0.00 \%)$ & $0(0.00 \%)$ & $576(54.14 \%)$ \\
\hline \multirow[t]{2}{*}{ N-stage } & No & $18(22.78 \%)$ & $24110(86.17 \%)$ & $6028(78.48 \%)$ & $5432(58.19 \%)$ & $721(36.27 \%)$ \\
\hline & N1 & $61(77.22 \%)$ & $3870(13.83 \%)$ & $1653(21.52 \%)$ & $3903(41.81 \%)$ & $1267(63.73 \%)$ \\
\hline \multirow[t]{2}{*}{ M-stage } & M0 & $60(71.43 \%)$ & $27992(99.73 \%)$ & $7643(98.91 \%)$ & $9137(97.46 \%)$ & $1612(76.51 \%)$ \\
\hline & M1 & $24(28.57 \%)$ & $75(0.27 \%)$ & $84(1.09 \%)$ & $238(2.54 \%)$ & $495(23.49 \%)$ \\
\hline \multirow[t]{5}{*}{ Radiation } & None or refused & $45(55.56 \%)$ & $18232(66.50 \%)$ & $2836(37.83 \%)$ & $2751(30.35 \%)$ & $699(34.13 \%)$ \\
\hline & Radiation Beam & $4(4.94 \%)$ & $108(0.39 \%)$ & $86(1.15 \%)$ & $155(1.71 \%)$ & $461(22.51 \%)$ \\
\hline & Radioisotopes & $31(38.27 \%)$ & $8940(32.61 \%)$ & $4498(60.00 \%)$ & $6037(66.61 \%)$ & $845(41.26 \%)$ \\
\hline & $\begin{array}{l}\text { Radioactive } \\
\text { implants }\end{array}$ & $1(1.23 \%)$ & $122(0.44 \%)$ & $70(0.93 \%)$ & $94(1.04 \%)$ & $14(0.68 \%)$ \\
\hline & $\begin{array}{l}\text { Radiation beam } \\
\text { +isotopes/implants }\end{array}$ & $0(0.00 \%)$ & $16(0.06)$ & $7(0.09 \%)$ & $26(0.29 \%)$ & $29(1.42 \%)$ \\
\hline \multirow[t]{3}{*}{ Surgery } & $\begin{array}{l}\text { Recommended but } \\
\text { not Performed }\end{array}$ & $2(2.38 \%)$ & $66(0.24 \%)$ & $34(0.44 \%)$ & $25(0.27 \%)$ & $28(1.34 \%)$ \\
\hline & Performed & $55(65.48 \%)$ & $27621(98.80 \%)$ & $7513(97.57 \%)$ & $9269(99.03 \%)$ & $1644(78.47 \%)$ \\
\hline & Not recommended & $27(32.14 \%)$ & $269(0.96 \%)$ & $153(1.99 \%)$ & $66(0.71 \%)$ & $423(20.19 \%)$ \\
\hline
\end{tabular}

after propensity score matching with age, race and tumor differentiation grade, $\mathrm{T} 0$ stage remained a poorer prognosis for cancer-specific mortality when compared to T1, T2 and T3 stage (Log-rank test, $p=0.0738,0.0698,0.0752$, respectively, Figure 2A, 2D and $2 \mathrm{G}$ ). After propensity score matching with age, race, tumor differentiation grade, $\mathrm{N}$ stage and $\mathrm{M}$ stage, T0 patients demonstrated a similar survival curve with T0-T3 patients (Figure 2B, 2E and $2 \mathrm{H}$ ). When all influential variables, including surgery and radiation treatment, were matched, T0 stage became a better prognosis for cancer-specific mortality, compared to T3 stage (Log-rank test, $p=0.0448$, Figure 2I). These findings verified our hypothesis that metastasis and less treatment compared to T1-T3 patients led to poorer prognosis in $\mathrm{T} 0$ patients.

\section{Risk factors for all-cause mortality and thyroid carcinoma-specific mortality in thyroid micro- carcinoma patients}

In 17,315 included micro-carcinoma patients, both univariate and multivariate Cox regression analyses showed that age, radiation beam and non-surgery were significant risk factors of all-cause death. Radioisotopes treatment was associated with better prognosis for all-cause mortality (Table 3). Multivariate Cox analysis suggested that lymph node metastasis was a risk factor of all-cause death in micro-carcinoma patients. For thyroid carcinomaspecific death, age and radiation beam treatment were demonstrated as risk factors. All 10 thyroid carcinomaspecific deaths in T1a patients received surgery. Among 
Table 2: Risk factors for survival: outcome is all-cause mortality and thyroid carcinoma specific mortality

\begin{tabular}{|c|c|c|c|c|c|c|c|c|c|}
\hline \multirow[b]{3}{*}{ Variables } & \multirow[b]{3}{*}{ level* } & \multicolumn{4}{|c|}{ All cause mortality } & \multicolumn{4}{|c|}{ Thyroid Carcinoma specific mortality } \\
\hline & & \multicolumn{2}{|c|}{ Univariate Cox regression } & \multicolumn{2}{|c|}{ Multivariate Cox regression } & \multicolumn{2}{|c|}{ Univariate Cox regression } & \multicolumn{2}{|c|}{ Multivariate Cox regression } \\
\hline & & $\begin{array}{l}\text { Hazard Ratio } \\
(95 \% \text { CI })\end{array}$ & $p$-value & $\begin{array}{l}\text { Hazard Ratio } \\
(95 \% \mathrm{CI})\end{array}$ & $p$-value & $\begin{array}{c}\text { Hazard Ratio } \\
(95 \% \mathrm{CI})\end{array}$ & $p$-value & $\begin{array}{l}\text { Hazard Ratio } \\
(95 \% \mathrm{CI})\end{array}$ & $p$-value \\
\hline Age & & $1.093(1.089,1.097)$ & $<0.0001$ & $1.054(1.049,1.058)$ & $<0.0001$ & $1.106(1.100,1.112)$ & $<0.0001$ & $1.042(1.035,1.048)$ & $<0.0001$ \\
\hline \multirow[t]{3}{*}{ Race } & White & ref & & ref & & ref & & ref & \\
\hline & Black & $1.287(1.080,1.533)$ & 0.005 & $1.324(1.091,1.606)$ & 0.005 & $1.189(0.912,1.550)$ & 0.20 & $1.269(0.942,1.709)$ & 0.12 \\
\hline & Other & $0.952(0.816,1.112)$ & 0.54 & $1.018(0.859,1.211)$ & 0.84 & $1.090(0.882,1.347)$ & 0.42 & $1.220(0.956,1.556)$ & 0.11 \\
\hline \multirow[t]{5}{*}{ T-stage } & T0 & ref & & Ref & & ref & & ref & \\
\hline & $\mathrm{T} 1$ & $0.125(0.065,0.243)$ & $<0.0001$ & $0.754(0.370,1.537)$ & 0.44 & $0.024(0.007,0.080)$ & $<0.0001$ & $0.146(0.034,0.622)$ & 0.009 \\
\hline & $\mathrm{T} 2$ & $1.177(0.090,0.347)$ & $<0.0001$ & $1.270(0.617,2.613)$ & 0.52 & $0.102(0.031,0.336)$ & 0.0002 & $0.799(0.188,3.388)$ & 0.76 \\
\hline & $\mathrm{T} 3$ & $0.244(0.126,0.475)$ & $<0.0001$ & $1.447(0.708,2.955)$ & 0.31 & $0.250(0.079,0.789)$ & 0.02 & $1.534(0.374,6.298)$ & 0.55 \\
\hline & $\mathrm{T} 4$ & $4.111(2.130,7.934)$ & $<0.0001$ & $3.882(1.909,7.895)$ & 0.0002 & $8.875(2.854,27.600)$ & 0.0002 & $7.236(1.777,29.463)$ & $<0.0001$ \\
\hline \multirow[t]{2}{*}{ N-stage } & No & ref & & Ref & & ref & & ref & \\
\hline & N1 & $2.564(2.313,2.842)$ & $<.0001$ & $1.371(1.207,1.558)$ & $<0.0001$ & $5.445(4.654,6.370)$ & $<.0001$ & $1.458(1.220,1.741)$ & $<0.0001$ \\
\hline \multirow[t]{2}{*}{ M-stage } & M0 & ref & & Ref & & ref & $<0.0001$ & Ref & \\
\hline & M1 & $\begin{array}{c}27.671 \\
(24.902,30.748)\end{array}$ & $<0.0001$ & $1.324(1.091,1.606)$ & $<0.0001$ & $\begin{array}{c}57.683(50.043, \\
66.489)\end{array}$ & & $2.705(2.250,3.252)$ & $<0.0001$ \\
\hline \multirow[t]{5}{*}{ Grade } & Well differentiated & ref & & Ref & & ref & & ref & \\
\hline & $\begin{array}{l}\text { Moderately } \\
\text { differentiated }\end{array}$ & $1.897(1.335,2.695)$ & 0.0004 & $1.337(0.934,1.912)$ & 0.11 & $2.321(0.882,6.106)$ & 0.09 & $1.393(0.505,3.848)$ & 0.52 \\
\hline & $\begin{array}{l}\text { Poorly } \\
\text { differentiated }\end{array}$ & $\begin{array}{c}24.298(18.972, \\
31.118)\end{array}$ & $<0.0001$ & $4.865(3.678,6.434)$ & $<0.0001$ & $\begin{array}{c}149.938 \\
(84.086,267.362)\end{array}$ & $<0.0001$ & $\begin{array}{c}16.443 \\
(8.401,32.183)\end{array}$ & $<0.0001$ \\
\hline & Undifferentiated & $\begin{array}{c}161.988 \\
(131.507,199.534)\end{array}$ & $<0.0001$ & $6.932(5.288,9.088)$ & $<0.0001$ & $\begin{array}{c}1148.482 \\
(660.170,1997.785)\end{array}$ & $<0.0001$ & $\begin{array}{c}24.513 \\
(12.598,47.696)\end{array}$ & $<0.0001$ \\
\hline & Unknown & $2.142(1.761,2.606)$ & $<0.0001$ & $1.387(1.128,1.706)$ & 0.002 & $5.657(3.241,9.871)$ & $<0.0001$ & $3.576(1.889,6.772)$ & $<0.0001$ \\
\hline \multirow[t]{5}{*}{ Radiation } & None or refused & ref & & ref & & ref & & ref & \\
\hline & Radiation Beam & $\begin{array}{c}13.924 \\
(12.341,15.709)\end{array}$ & $<0.0001$ & $0.803(0.658,9.943)$ & 0.07 & $\begin{array}{c}27.967(24.015 \\
32.569)\end{array}$ & $<0.0001$ & $0.777(0.643,0.939)$ & 0.009 \\
\hline & Radioisotopes & $0.266(0.230,0.307)$ & $<0.0001$ & $0.342(0.292,0.401)$ & $<0.0001$ & $0.202(0.156,0.261)$ & $<0.0001$ & $0.244(0.184,0.325)$ & $<0.0001$ \\
\hline & $\begin{array}{l}\text { Radioactive } \\
\text { implants }\end{array}$ & $0.473(0.212,1.055)$ & 0.07 & $0.556(0.248,1.243)$ & 0.15 & $0.407(0.102,1.635)$ & 0.21 & $0.402(0.100,1.621)$ & 0.20 \\
\hline & $\begin{array}{l}\text { Radiation beam } \\
\text { +isotopes/ } \\
\text { implants }\end{array}$ & $2.775(1.440,5.346)$ & 0.002 & $0.645(0.331,1.259)$ & 0.20 & $6.147(3.052,12.378)$ & $<0.0001$ & $0.805(0.393,1.651)$ & 0.55 \\
\hline \multirow[t]{3}{*}{ Surgery } & $\begin{array}{l}\text { Recommended but } \\
\text { not Performed }\end{array}$ & ref & & ref & & ref & & ref & \\
\hline & Performed & $0.060(0.046,0.077)$ & $<0.0001$ & $0.352(0.252,0.491)$ & $<0.0001$ & $0.040(0.028,0.057)$ & $<0.0001$ & $0.497(0.311,0.795)$ & 0.004 \\
\hline & Not recommended & $2.021(1.555,2.627)$ & $<0.0001$ & $1.295(0.927,1.811)$ & 0.13 & $2.335(1.643,3.319)$ & $<0.0001$ & $1.350(0.851,2.143)$ & 0.20 \\
\hline
\end{tabular}

9 deaths whose radiation treatment information was known, 7 did not receive any radiation therapy (Table 3).

\section{Survival analysis among thyroid micro- carcinoma patients}

Unlike T0 patients $(65.5 \%$ received surgery and $44.4 \%$ received radiation), $99.3 \%$ T1a patients received surgery, while only $21.46 \%$ patients received radiation therapy. In thyroid micro-carcinoma patients, the 1-, 2- and 4-year estimated survival rate was $99.32 \%$, $98.64 \%$ and $97.50 \%$, respectively (Supplementary Figure S4A). The 1-, 2- and 4-year thyroid carcinomaspecific estimated survival rate was $99.95 \%, 99.93 \%$ and $99.92 \%$, respectively (Supplementary Figure S4B). Kaplan Meier curves showed no significant difference in cancer-specific survival stratified by surgery (log rank $p=0.82)$ and radiation therapy $(\log \operatorname{rank} p=0.96)$
(Figure 3). The survival rate information stratified by surgery and radiation therapy was demonstrated in Supplementary Tables S3 and S4.

\section{DISCUSSION}

Overdiagnosis and overtreatment problem are common in thyroid carcinoma [12]. On the other hand, surgeons and oncologists always ignored T0 patients due to perceived low mortality rate [5]. Our findings showed that compared to T1-T3 patients (>97\% patients underwent surgery), only 65\% T0 patients received surgery. Compared to $\mathrm{T} 2-\mathrm{T} 4$ patients $(>60 \%$ patients underwent radiation therapy), only about $44 \% \mathrm{~T} 0$ patients received radiotherapy (Table 1).

Based on SEER data 2011-2013, T0 stage had a significantly poorer thyroid carcinoma-specific survival and all-cause survival compared to T1-T3 patients 
(Figure 1). More metastasis and less treatment compared to T1-T3 patients were the two major reasons. For example, compared to T3 patients (41.81\% lymph node metastasis and $2.54 \%$ distant metastasis), $77.22 \%$ T0 patients had lymph node metastasis and $28.57 \%$ had distant metastasis. But, only $65.48 \%$ and $44.44 \%$ T0 patients received surgery and radiation therapy, significantly lower than $\mathrm{T} 3$ patients (99.03\% received surgery and $69.65 \%$ received radiation) (Table 1).

Propensity score matching analysis further verified our hypothesis. As shown in figure 2, T0 patients still showed marginally lower survival rate when age, race and histology grade were matched with T1-T3 patients (Log-rank test, $p=0.0738,0.0698,0.0752$, respectively, Figure 2A, 2D and 2G). When $\mathrm{N}$ stage and $\mathrm{M}$ stage were matched, T0 patients showed similar survival curve with T1-T3 patients. This finding suggests that late-stage in terms of nodes or metastases lead to poorer prognosis in T0 patients. Moreover, when radiation and surgery treatment were matched, T0 patients had significantly higher survival compared to $\mathrm{T} 3$ patients. This means that if $\mathrm{T} 0$ patients had the same metastasis stage and treatment with T3 patients, T0 patients would have a better prognosis.

As for the possible mis-staged problem in T0 patients, the chance is small. SEER database records actual primary tumor size information in column " $\mathrm{CS}$ tumor size 2004+", and records T stage information in column "Derived AJCC T (7th ed, 2010)". Since T stages were defined based on primary thyroid tumor sizes, the patients' $T$ stages were consistent to the corresponding actual primary tumor sizes in this study.

Among thyroid micro-carcinoma patients, even the 4-year survival rate was 99.92\% (Supplementary Figure S4), still more than $99 \%$ patients received surgery, and $21.55 \%$ patients received both surgery and radiation therapies. Thyroid carcinoma-specific survival analysis suggested that no survival difference was observed in micro-carcinoma patients stratified by surgery or radiation therapy (Figure 3, Supplementary Table S3 and S4). Multivariate Cox regression analysis showed that, by controlling other factors, radiation beam therapy was a risk factor of all-cause mortality with a hazard ratio of 3.713 (95\% CI: [1.154, 11.948], Table 3). Radioisotopes and surgery strategies cause irreparable life-long damages to human organs and lead to several morbidities, including hypothyroidism, salivary gland disturbance and vocal disorders [13-19]. Recommendation for nonsurgical treatment strategy includes ultrasound-guided thermal laser ablation (LA). It is safe, repeatable and works well for tumors less than $15 \mathrm{~mm}$ [20-21]. This nonsurgical and repeatable strategy would be promising in future.

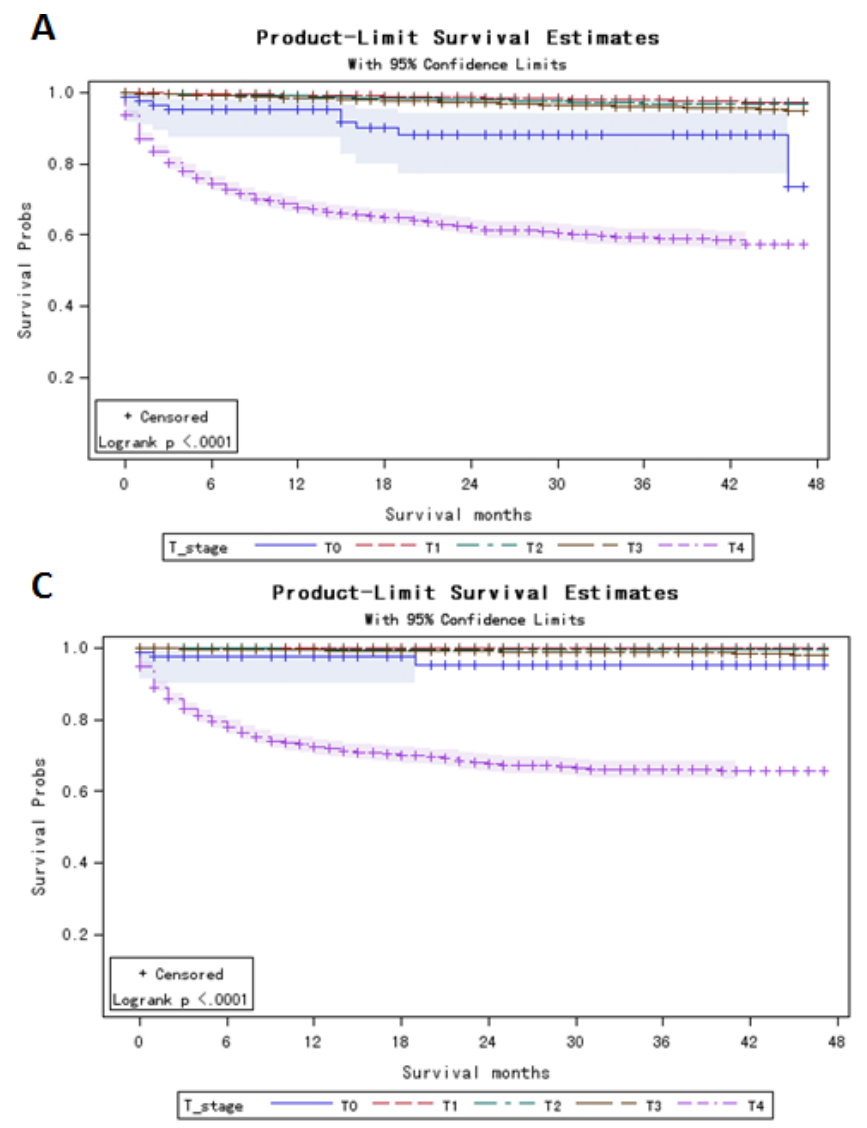

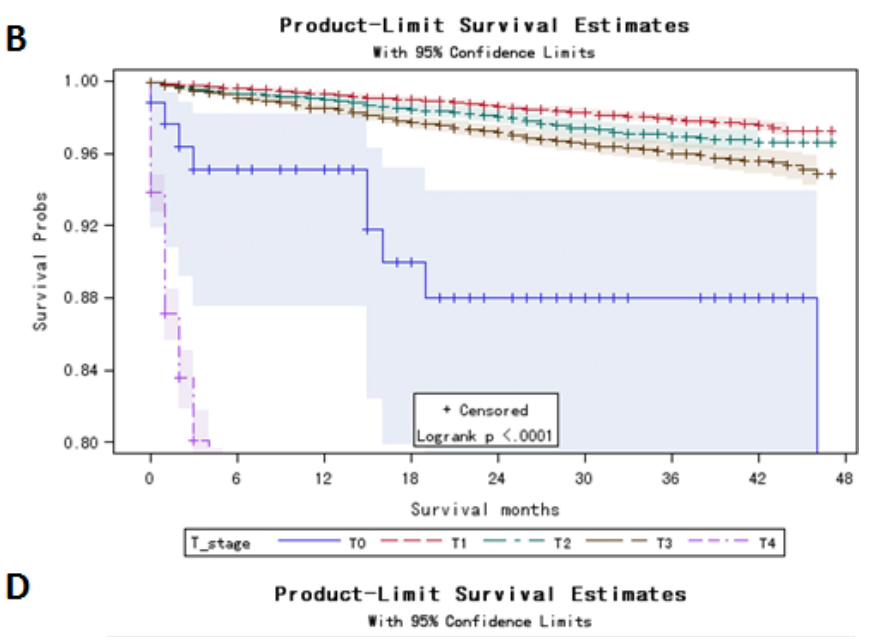

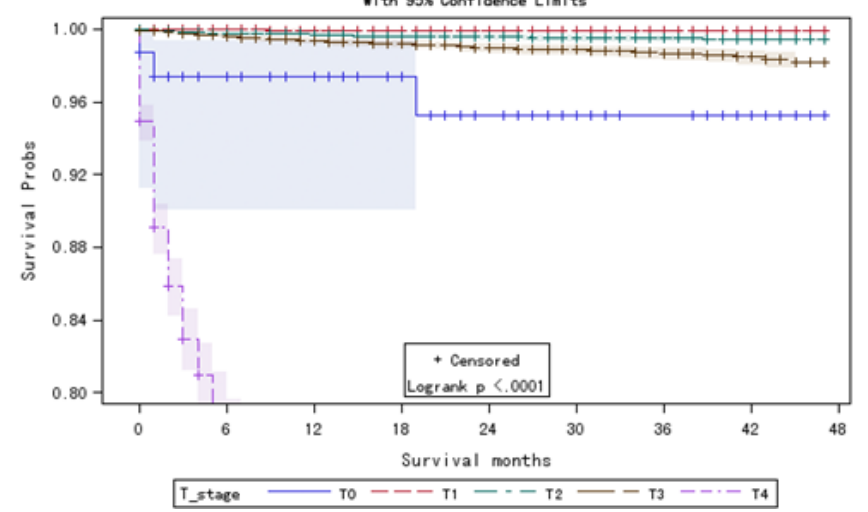

Figure 1: Kaplan Meier curves among patients stratified by T-stage for all cause mortality (A, B: Log rank test $p<0.0001)$ and thyroid carcinoma-specific mortality $(C, D:$ Log rank test $p<0.0001)$ 
Table 3: Risk factors for survival in patients with micro thyroid carcinoma patients: outcome is all cause mortality $(n=17,587)$ and thyroid carcinoma-specific mortality $(n=17,315)$

\begin{tabular}{|c|c|c|c|c|c|c|c|c|c|c|c|}
\hline \multirow{3}{*}{ Variables } & \multirow{3}{*}{ level * } & \multicolumn{5}{|c|}{ All cause mortality } & \multicolumn{5}{|c|}{ Thyroid Carcinoma specific mortality } \\
\hline & & \multirow{2}{*}{$N^{* *}$} & \multicolumn{2}{|c|}{ Univariate Cox regression } & \multicolumn{2}{|c|}{$\begin{array}{c}\text { Multivariate Cox } \\
\text { regression }\end{array}$} & \multirow[b]{2}{*}{$N^{* *}$} & \multicolumn{2}{|c|}{$\begin{array}{c}\text { Univariate Cox } \\
\text { regression }\end{array}$} & \multicolumn{2}{|c|}{$\begin{array}{c}\text { Multivariate Cox } \\
\text { regression }\end{array}$} \\
\hline & & & $\begin{array}{l}\text { Hazard Ratio (95\% } \\
\text { CI) }\end{array}$ & $p$-value & $\begin{array}{c}\text { Hazard Ratio } \\
(95 \% \text { CI })\end{array}$ & $p$-value & & $\begin{array}{c}\text { Hazard Ratio } \\
(95 \% \text { CI })\end{array}$ & $p$-value & $\begin{array}{c}\text { Hazard Ratio } \\
(95 \% \text { CI })\end{array}$ & $p$-value \\
\hline Age & & & $1.076(1.064,1.087)$ & $<0.0001$ & $\begin{array}{c}1.074 \\
(1.063,1.085)\end{array}$ & $<0.0001$ & & $\begin{array}{c}1.066 \\
(1.015,1.119)\end{array}$ & 0.008 & $\begin{array}{c}1.069 \\
(1.015,1,125)\end{array}$ & 0.01 \\
\hline \multirow[t]{3}{*}{ Race } & White & $227 / 14420$ & ref & & ref & & $7 / 14200$ & ref & & ref & \\
\hline & Black & $34 / 1267$ & $1.336(0.851,2.099)$ & 0.21 & $\begin{array}{c}1.447 \\
(0.919,2.277)\end{array}$ & 0.11 & $1 / 1234$ & $\begin{array}{c}1.652 \\
(0.203,13.424)\end{array}$ & 0.64 & $\begin{array}{c}2.244 \\
(0.264,19.052)\end{array}$ & 0.46 \\
\hline & Other & $21 / 1900$ & $0.841(0.525,1.350)$ & 0.47 & $\begin{array}{c}1.008 \\
(0.626,1.622)\end{array}$ & 0.97 & $2 / 1881$ & $\begin{array}{c}2.258 \\
(0.469,10.868)\end{array}$ & 0.31 & $\begin{array}{c}3.155 \\
(0.630,15.813)\end{array}$ & 0.16 \\
\hline \multirow[t]{5}{*}{ Grade } & $\begin{array}{l}\text { Well } \\
\text { differentiated }\end{array}$ & $34 / 3759$ & ref & & ref & & $2 / 3727$ & ref & & ref & \\
\hline & $\begin{array}{l}\text { Moderately } \\
\text { differentiated }\end{array}$ & $5 / 432$ & $1.309(0.511,3.353)$ & 0.57 & $\begin{array}{c}1.073 \\
(0.418,2.753)\end{array}$ & 0.88 & $0 / 427$ & $0(0,)$. & 0.99 & $0(0,)$. & 1.00 \\
\hline & $\begin{array}{l}\text { Poorly } \\
\text { differentiated }\end{array}$ & $1 / 35$ & $3.119(0.428,22.725)$ & 0.26 & $\begin{array}{c}2.146 \\
(0.292,15.779)\end{array}$ & 0.45 & $0 / 34$ & $0(0,)$. & 0.00 & $0(0,)$. & 1.00 \\
\hline & Undifferentiated & $0 / 0$ & - & & - & & $0 / 0$ & - & & - & \\
\hline & Unknown & $242 / 13361$ & $1.452(1.002,2.103)$ & 0.05 & $\begin{array}{c}1.280 \\
(0.881,1.860)\end{array}$ & 0.20 & $8 / 13127$ & $\begin{array}{c}1.080 \\
(0.229,5.086)\end{array}$ & 0.92 & $\begin{array}{c}1.021 \\
(0.206,5.052)\end{array}$ & 0.98 \\
\hline \multirow[t]{2}{*}{ N-stage } & No & $254 / 15882$ & ref & & ref & & $8 / 15636$ & ref & & ref & \\
\hline & N1 & $27 / 1651$ & $1.323(0.855,1.979)$ & 0.17 & $\begin{array}{c}2.380 \\
(1.524,3.718)\end{array}$ & 0.0001 & $2 / 1626$ & $\begin{array}{c}2.397 \\
(0.509,11.286)\end{array}$ & 0.27 & $\begin{array}{c}4.246 \\
(0.638,28.274)\end{array}$ & 0.13 \\
\hline \multirow[t]{2}{*}{ M-stage } & M0 & $281 / 17556$ & ref & & ref & & $10 / 17285$ & ref & & ref & \\
\hline & M1 & $1 / 31$ & $2.350(0.330,16.758)$ & 0.39 & $\begin{array}{c}1.680 \\
(0.230,12.270)\end{array}$ & 0.61 & $0 / 30$ & $0(0,)$. & 1.00 & $0(0,)$. & 1.00 \\
\hline \multirow[t]{5}{*}{ Radiation } & None or refused & $242 / 13531$ & ref & & ref & & $7 / 13296$ & ref & & ref & \\
\hline & Radiation Beam & $3 / 43$ & $4.927(1.574,15.423)$ & 0.006 & $\begin{array}{c}3.713 \\
(1.154,11.948)\end{array}$ & 0.03 & $1 / 41$ & $\begin{array}{c}46.612 \\
(5.734,378.880)\end{array}$ & 0.0003 & $\begin{array}{c}24.597 \\
(2.114,286.227)\end{array}$ & 0.01 \\
\hline & Radioisotopes & $33 / 3598$ & $0.600(0.414,0.870)$ & 0.007 & $\begin{array}{c}0.653 \\
(0.434,0.981)\end{array}$ & 0.04 & $1 / 3566$ & $\begin{array}{c}0.494 \\
(0.061,4.016)\end{array}$ & 0.51 & $\begin{array}{c}0.388 \\
(0.040,3.770)\end{array}$ & 0.41 \\
\hline & $\begin{array}{l}\text { Radioactive } \\
\text { implants }\end{array}$ & $1 / 52$ & $1.483(0.208,10.583)$ & 0.69 & $\begin{array}{c}1.599 \\
(0.222,11.542)\end{array}$ & 0.64 & $0 / 51$ & $0(0,)$. & 1.00 & $0(0,)$. & 1.00 \\
\hline & $\begin{array}{l}\text { Radiation beam } \\
\text { +isotopes/ } \\
\text { implants }\end{array}$ & $0 / 5$ & $0(0, \mathrm{I})$ & 0.98 & $0(0, \mathrm{I})$ & 0.97 & $0 / 5$ & $0(0,)$. & 1.00 & $0(0,)$. & 1.00 \\
\hline \multirow[t]{3}{*}{ Surgery } & $\begin{array}{l}\text { Recommended } \\
\text { but not } \\
\text { Performed }\end{array}$ & $4 / 33$ & ref & & ref & & $0 / 29$ & ref & & ref & \\
\hline & Performed & $207 / 17390$ & $0.081(0.030,0.217)$ & $<0.0001$ & $\begin{array}{c}0.119 \\
(0.038,0.373)\end{array}$ & 0.0003 & $10 / 17193$ & $\mathrm{I}(0,)$. & 1.00 & $\mathrm{I}(0,)$. & 1.00 \\
\hline & $\begin{array}{l}\text { Not } \\
\text { recommended }\end{array}$ & $10 / 90$ & $1.006(0.316,3.209)$ & 0.99 & $\begin{array}{c}1.035 \\
(0.284,3.770)\end{array}$ & 0.96 & $0 / 80$ & $1.00(0,)$. & 1.00 & $0.728(0,)$. & 1.00 \\
\hline
\end{tabular}

Note: $N^{* *}$ is demonstrated as numerator divided by denominator, where numerator indicates the number of death and denominator indicates the number of patients in each category level for every variable

\section{MATERIALS AND METHODS}

\section{Database}

The SEER*Stat database, which was released by the Surveillance Research Program at the National Cancer Institute (NCI) in 2016, was used as the data source in the present study [22]. 48,234 patients diagnosed as thyroid carcinoma (ICCC site recode ICD-O-3/WHO 2008 and Behavior code ICD-O-3: malignant) were identified in the SEER 18 Research Data + Hurricane Katrina Impacted Louisiana Cases, Nov 2015 Sub (1973-2013 varying) incidence database. Because SEER database records detailed T stage (AJCC 2010) information from 2010, so we only included cases diagnosed from 2010 to 2013.

\section{AJCC T staging}

To compare the survival rate among different T stages, 47,360 patients were categorized according to AJCC T staging (2010). SEER database records the actual tumor size. T stages were defined based on primary thyroid tumor size. In this study, the $\mathrm{T}$ stages were consistent to the corresponding actual tumor size.

Demographics, lymph node metastasis, distant metastasis, differentiation grade, surgery treatment 
A

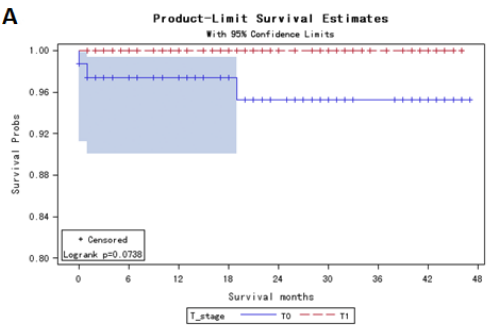

D

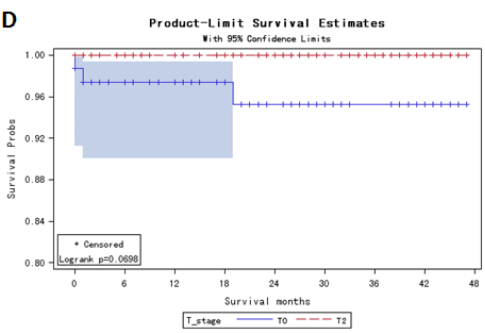

G

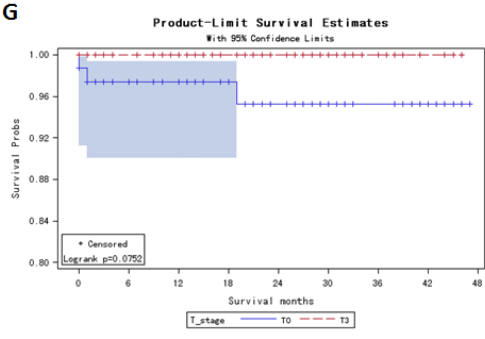

J

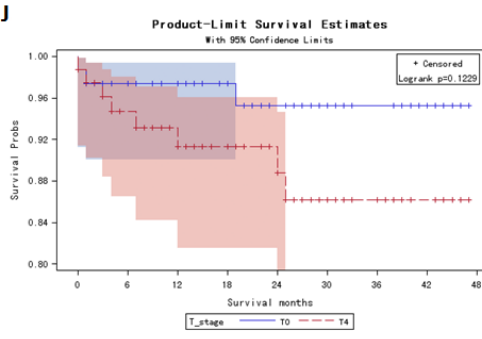

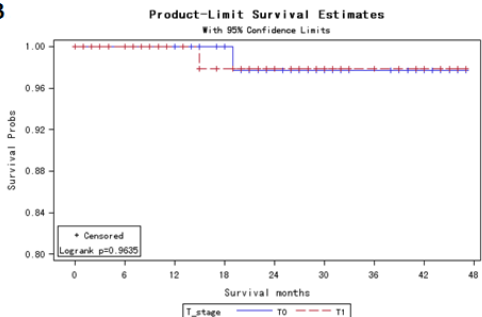

E
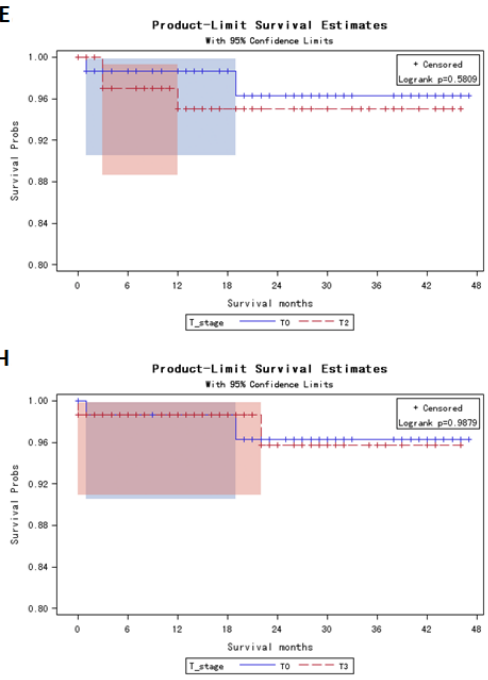

K

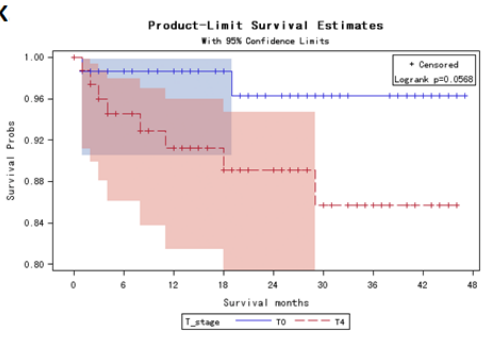

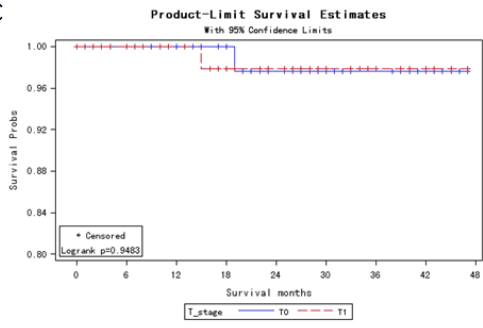

$\mathbf{F}$
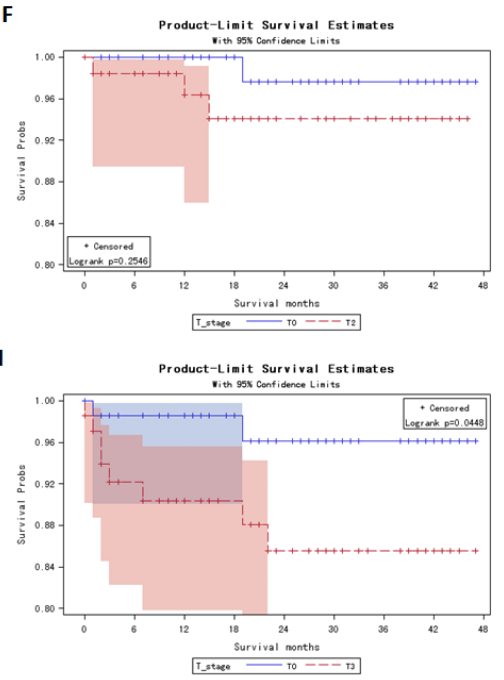

$\mathbf{L}$

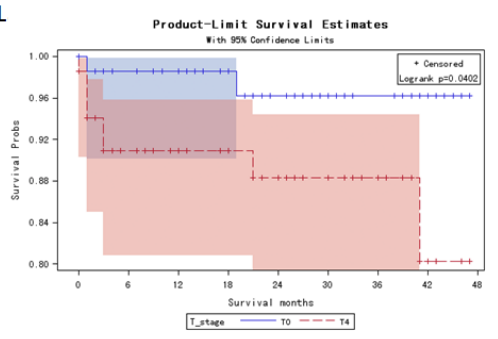

Figure 2: Kaplan Meier curves for matched T-stage pairs. Age, race and differentiation grade matching between T0 and T1 (A), T0 and T2 (D), T0 and T3 (G), T0 and T4 (J). Age, race, differentiation grade, N / M stage matched between T0 and T1 (B), T0 and T2 (E), T0 and T3 (H), T0 and T4 (K). Age, race, differentiation grade, N / M stage, surgery and radiation treatment matched between T0 and T1 (C), T0 and T2 (F), T0 and T3 (I), T0 and T4 (L).

A

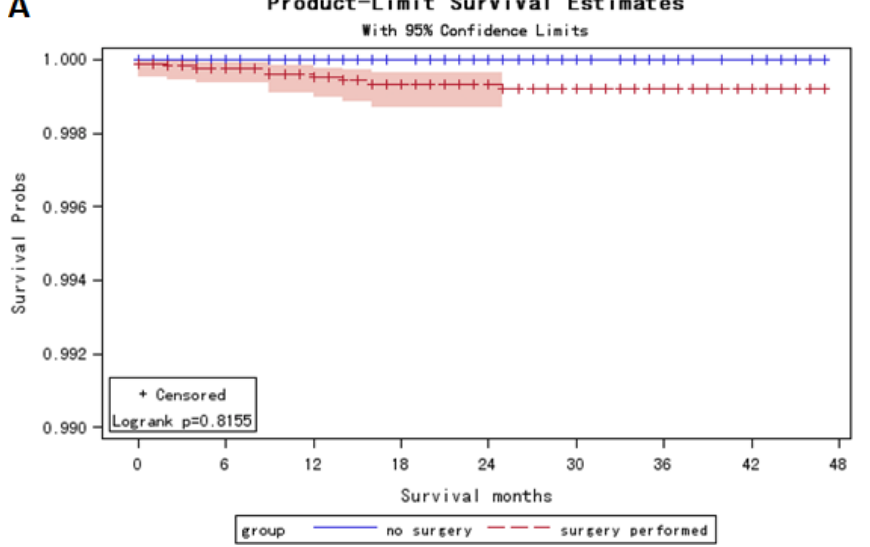

B

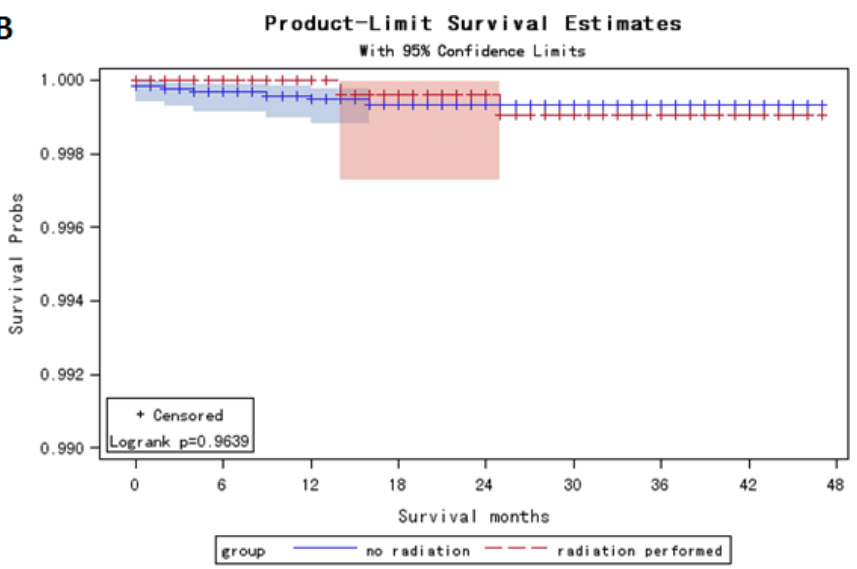

Figure 3: Kaplan Meier curves among thyroid micro-carcinoma patients for thyroid carcinoma specific mortality and thyroid carcinoma-specific mortality stratified by surgery treatment (A Log rank test $p=0.82)$ and radiation treatment (B Log rank test $p=0.96)$. 
(surgery performed, recommended but not performed, and not recommended) and radiation treatment (radioisotopes, beam radiation, radioactive implants, combination of beam with implants or isotopes, and none) were evaluated in patients with different $\mathrm{T}$ categories. To investigate the treatment efficiency in thyroid micro-carcinoma (T1a) patients, 17,315 T1a patients were categorized based on surgery and radiation treatment types. Survival analysis was performed to evaluate the treatment efficiency.

\section{Statistical analysis}

Patients were followed up until December 2013. The primary outcome measures thyroid carcinoma-specific mortality. The secondary outcome measures the all-cause mortality. The candidate risk factors included age, race, differentiation grade, surgery type, radiation type, and TNM stage. Numeric variables were summarized as the mean (standard deviation) or median (interquartile range), where appropriate. Categorical variables were reported as counts (percentage). An analysis of variance was used to compare continuous variables with symmetric distributions among different treatment types and T staging categories. Chi-square tests or Fisher's exact tests $(n<5)$ were used to compare categorical variables among $\mathrm{T}$ staging categories or treatment group. The Kaplan-Meier method was used to plot the survival distributions, and the log-rank test was used to assess differences in survival experience between the groups. The Cox proportional hazards regression was performed to estimate the hazard ratio to identify the risk factors for thyroid carcinoma-specific mortality and allcause mortality. To further adjust for potential baseline confounders, a propensity score matching was carried out. A receiver operating characteristic (ROC) curve was also calculated to determine the optimal cutoff that maximizes sensitivity and specificity in predicting mortality. All tests of hypotheses were two-tailed and conducted at a significance level of 0.05 . Statistical analyses were conducted using SAS 9.4.

\section{ACKNOWLEDGMENTS}

This study used the SEER 18 Regs research database as the data source. The interpretation and reporting of these data are the sole responsibility of the authors.

The authors acknowledge the efforts of the National Cancer Institute; the Surveillance, Epidemiology, and End Results (SEER) Program tumor registry; and the Information Management Service Inc. for the creation and distribution of the SEER*Stat database.

\section{CONFLICTS OF INTEREST}

None.

\section{FUNDING}

None.

\section{Authors' contributions}

Dr. Hu had full access to all of the data in the study and took responsibility for the integrity and accuracy of the data analysis. Study concept and design: Zhou and Tian. Acquisition, analysis or interpretation of the data: Zhou, Tian, Li and Hu Drafting of the manuscript: Hu. Critical revision of the manuscript for important intellectual content: All authors. Statistical analysis: $\mathrm{Hu}$ Study supervision: Zhou and Hu.

\section{REFERENCES}

1. Davies L, Welch HG. Increasing incidence of thyroid cancer in the United States, 1973-2002. JAMA. 2006; 295:2164-7. doi: 295/18/2164 [pii]10.1001/jama.295.18.2164.

2. Davies L, Welch HG. Thyroid cancer survival in the United States: observational data from 1973 to 2005. Arch Otolaryngol Head Neck Surg. 2010; 136:440-4. doi: 136/5/440 [pii]10.1001/archoto.2010.55.

3. Jarzab B, Dedecjus M, Handkiewicz-Junak D, Lange D, Lewinski A, Nasierowska-Guttmejer A, Ruchala M, Slowinska-Klencka D, Nauman J, Adamczewski Z, Baglaj M, Baldys-Waligorska A, Barczynski M, et al. Diagnostics and Treatment of Thyroid Carcinoma. Endokrynol Pol. 2016; 67:74-107. doi: VM/OJS/J/44064 [pii]10.5603/EP.2016.0011.

4. Hay ID, Grant CS, Bergstralh EJ, Thompson GB, van Heerden JA, Goellner JR. Unilateral total lobectomy: is it sufficient surgical treatment for patients with AMES low-risk papillary thyroid carcinoma? Surgery. 1998; 124:958-64. discussion 64-6. doi: S0039606098003717 [pii].

5. Sarda AK, Aggarwal S, Pandey D, Gautam G. Prognostic factors for well-differentiated thyroid cancer in an endemic area. Asian J Surg. 2002; 25:325-9. doi: S10159584(09)60200-1 [pii]10.1016/S1015-9584(09)60200-1.

6. Lin HW, Bhattacharyya N. Survival impact of treatment options for papillary microcarcinoma of the thyroid. Laryngoscope. 2009; 119:1983-7. doi: 10.1002/lary.20617.

7. Anderson KL, Jr., Youngwirth LM, Scheri RP, Stang MT, Roman SA, Sosa JA. T1a Versus T1b Differentiated Thyroid Cancers: Do We Need to Make the Distinction? Thyroid. 2016; 26:1046-52. doi: 10.1089/thy.2016.0073.

8. Lang BH, Wong CK. Lobectomy is a more Cost-Effective Option than Total Thyroidectomy for 1 to $4 \mathrm{~cm}$ Papillary Thyroid Carcinoma that do not Possess Clinically Recognizable High-Risk Features. Ann Surg Oncol. 2016; 23:3641-52. doi: 10.1245/s 10434-016-5280-610.1245/ s10434-016-5280-6 [pii]. 
9. Gulcelik MA, Kuru B, Dincer H, Camlibel M, Yuksel UM, Yenidogan E, Reis E. Complications of completion versus total thyroidectomy. Asian Pac J Cancer Prev. 2012; 13:5225-8. doi.

10. Valcavi R, Piana S, Bortolan GS, Lai R, Barbieri V, Negro R. Ultrasound-guided percutaneous laser ablation of papillary thyroid microcarcinoma: a feasibility study on three cases with pathological and immunohistochemical evaluation. Thyroid. 2013; 23:1578-82. doi: 10.1089/ thy.2013.0279.

11. Papini E, Guglielmi R, Gharib H, Misischi I, Graziano F, Chianelli M, Crescenzi A, Bianchini A, Valle D, Bizzarri G. Ultrasound-guided laser ablation of incidental papillary thyroid microcarcinoma: a potential therapeutic approach in patients at surgical risk. Thyroid. 2011; 21:917-20. doi: 10.1089/thy.2010.0447.

12. Lubitz CC, Sosa JA. The changing landscape of papillary thyroid cancer: Epidemiology, management, and the implications for patients. Cancer. 2016. doi: 10.1002/ cncr.30201.

13. Alba JR, Basterra J, Ferrer JC, Santonja F, Zapater E. Hypothyroidism in patients treated with radiotherapy for head and neck carcinoma: standardised long-term follow-up study. J Laryngol Otol. 2016; 130:478-81. doi: S0022215116000967 [pii]10.1017/S0022215116000967.

14. Cetinayak O, Akman F, Kentli S, Duzen M, Eyiler F, Sen M, Kinay M. Assessment of treatment-related thyroid dysfunction in patients with head and neck cancer. Tumori. 2008; 94:19-23.

15. Jeong SY, Kim HW, Lee SW, Ahn BC, Lee J. Salivary gland function 5 years after radioactive iodine ablation in patients with differentiated thyroid cancer: direct comparison of pre- and postablation scintigraphies and their relation to xerostomia symptoms. Thyroid. 2013; 23:609-16. doi: 10.1089/thy.2012.0106.

16. Raza H, Khan AU, Hameed A, Khan A. Quantitative evaluation of salivary gland dysfunction after radioiodine therapy using salivary gland scintigraphy. Nucl Med Commun. 2006; 27:495-9. doi: 00006231-20060600000004 [pii].

17. Roy N, Merrill RM, Gray SD, Smith EM. Voice disorders in the general population: prevalence, risk factors, and occupational impact. Laryngoscope. 2005; 115:1988-95. doi: 00005537-200511000-00016 [pii]10.1097/01. mlg.0000179174.32345.41.

18. Krausz Y, Uziely B, Nesher R, Chisin R, Glaser B. Recombinant thyroid-stimulating hormone in differentiated thyroid cancer. Isr Med Assoc J. 2001; 3:843-9.

19. Ha EJ, Baek JH, Lee JH. The efficacy and complications of radiofrequency ablation of thyroid nodules. Curr Opin Endocrinol Diabetes Obes. 2011; 18:310-4. doi: 10.1097/ MED.0b013e32834a9168.

20. Mauri G, Cova L, Ierace T, Baroli A, Di Mauro E, Pacella CM, Goldberg SN, Solbiati L. Treatment of Metastatic Lymph Nodes in the Neck from Papillary Thyroid Carcinoma with Percutaneous Laser Ablation. Cardiovasc Intervent Radiol. 2016; 39:1023-30. doi: 10.1007/s00270016-1313-610.1007/s00270-016-1313-6 [pii].

21. Zhou W, Zhang L, Zhan W, Jiang S, Zhu Y, Xu S. Percutaneous laser ablation for treatment of locally recurrent papillary thyroid carcinoma $<15 \mathrm{~mm}$. Clin Radiol. 2016. doi: S0009-9260(16)30297-5 [pii]10.1016/j. crad.2016.07.010.

22. Software. Surveillance Research Program, National Cancer Institute SEER*Stat software. wwwseercancergov/seerstat. 2016; version 8.3.2. doi. 